\title{
Significance of Visual Interfaces in Institutional and User-Generated Databases with Category Structures
}

\author{
Almila Akdag Salah \\ New Media Studies, Univ. of \\ Amsterdam, the Netherlands \\ and Sabancı Univ., Turkey \\ a.a.akdag@uva.nl \\ Peter Doorn \\ DANS, KNAW, the \\ Netherlands \\ peter.doorn@dans.knaw.nl
}

\author{
Andrea Scharnhorst \\ DANS, KNAW, the \\ Netherlands
}

first.lastname@dans.knaw.nl

\author{
Olav Ten Bosch \\ Drastic Data, the Netherlands \\ info@drasticdata.nl
}

\author{
Lev Manovich \\ Software Studies. UCSD, USA \\ manovich.lev@gmail.com
}

\author{
Albert Ali Salah \\ Dept. Computer Eng. \\ Boğaziçi Univ., Turkey \\ salah@boun.edu.tr
}

\begin{abstract}
In this paper we visually explore the data structure of two different visual platforms: the database behind the social environment of a social networking site, and the intricate infrastructure of a research institute for preservation of deposited datasets. We argue that visual analytics of metadata of collections can be used in multiple ways: for the backend users, to inform the archive about structure and growth of its collection; to foster collection strategies; and to check metadata consistency, for the end-users, to give an overview to the collections, and thus to generate more awareness of the collection and its metadata, to give the enduser extra information to contextualize the entirety of the archive. We conclude with a discussion on how text based search combined with different type of visually enhanced browsing improves data access, navigation, and reuse in these two radically different contexts.
\end{abstract}

\section{Categories and Subject Descriptors}

H.5.2 [User Interfaces]: Graphical User Interfaces; D.2.2 [User Interfaces]: D.2.2 [User Interfaces], H.5.2 User Interfaces, Graphical User Interfaces

\section{Keywords}

Visual interfaces to digital collections, archive, metadata, categories and classifications, tree map, visual faceted browsing, user-generated collections, social network sites, deviantArt.

\section{INTRODUCTION}

Visualization of data is a high craft, and the last decade has seen a movement in popularizing and democratizing vi-

Permission to make digital or hard copies of all or part of this work for personal or classroom use is granted without fee provided that copies are not made or distributed for profit or commercial advantage and that copies bear this notice and the full citation on the first page. To copy otherwise, to republish, to post on servers or to redistribute to lists, requires prior specific permission and/or a fee.

PATCH' 12, November 2, 2012, Nara, Japan.

Copyright 2012 ACM 978-1-4503-1594-4/12/11 ...\$15.00. sualization methods, and the introduction of interactive visual analytics. Today, a large variety of visualization modules and tools are freely available. However, when it comes to interactively visualizing large data collections, the available tools fall short. A customized search interface that fits the specifics of the digital archive is necessary. It may not be possible to keep such an interactive visualization completely up to date, because of the sheer size of the data, but this may not be necessary: the purpose of an overview is to give a notion of the dataset dynamics in an abstract way.

In the age of digital libraries, OPAC's (Online Public Access Catalogue) determine how the user engages with the collection of a library or an archive [3]. Retrieval typically produces lists of hits, and sometimeswith some statistical information - such as overall number of hits, or hits in a certain subject category - but only rarely visual exploration of the databases is given to the users as a choice.

In this paper we argue that the standard searching and browsing features hide essential information from the endusers, for example such as the category structure, which in essence helps the users to visualize the overview of the collection, as well as to contextualize a direct hit by showing the result within this overview, or the network structure, which enables the user to spatialize the collection, and to navigate within it.

There is a large body of literature on new interfaces for digital libraries and collections [4]. For some collections, principles as tag clouds ${ }^{1}$ [8], network visualizations ('Aquabrowser') ${ }^{2}$ [5], or concept browsing ${ }^{3}$ (The National Digital Library of the US) have been implemented into search interfaces. Shneiderman et al. [7] developed a visualization software for digital collections, applying his now famous principle of 'overview first, zoom and filter, then details-on demand' [7], combining category structure and its hierarchy to create the so-called hiaraxes. Yet social networks may have different needs. For example, the Vizster program [6] disregards Shneiderman's principle, arguing that each member in the network would be more interested in their immediate neighborhood, and would be intimidated by a complicated

\footnotetext{
${ }^{1}$ An example is the use of a tag cloud in http://www.librarything.com for navigation.

${ }^{2}$ http://zoeken.oba.nl/?q=garden $\& x=0 \& y=0$

${ }^{3}$ http://strandmaps.nsdl.org/
} 
network layout. This argument holds well for Friendster, the SNS originally analyzed with Vizster, which is a dating site [6].

Inspired by such experiments, we explore visual interfaces to two different collections in this paper: a dump of the DANS (Data Archiving and Networked Services) archive EASY $^{4}$, the largest national research database archive of the Netherlands, and a dataset derived from deviantArt ${ }^{5}$, the largest social networking site (SNS) on user-generated artworks. At first look, these two sites have nothing in common, beside the purpose of making large amounts of data available for their end users. DANS, armed with a researchbased perspective, has obligations to all researchers who are using its services to deposit their own datasets, as well as to a wider public (of researchers) who make use of these datasets for scientific exploration. deviantArt needs to offer a better search experience to its members, in order to compete in the commercial venue. However, both enterprises have the same basic search browser to access their database, and both possess an informative category structure which we seek to leverage in this paper.

This is a work in progress with the long-term goal to develop visual browsing tools enhancing search and navigation. Our contributions are 1) an analysis of two different case studies with different interaction needs, 2) interactive visualizations for each of these case studies, and 3) a discussion on how to improve access to large amounts of data. Each of our two case studies are devoted a section. For each case study, we describe the role and (institutional) background of our data sources, and describe our data. We then comment on the visual practices used, and how they are applied to the datasets, and briefly analyze the results. We conclude with a discussion of visualization of databases for different purposes and end-users.

\section{CASE STUDY 1: DANS-EASY}

\subsection{The self-archiving system EASY of DANS}

DANS is the largest national research data archive in the Netherlands in the social sciences and humanities. It is a public institution founded in 2005 by the Netherlands Organization for Scientific Research (NWO), and the Royal Netherlands Academy of Arts and Sciences (KNAW). Among its ancestors were economic-historical and sociological archives from the 60s. This long history of a relatively new institution explains some specificity of the metadata. The list of fields or categories used to index datasets is a subset of a disciplinary classification system designed for the Dutch Research Information System (NOD) in the 1980s, and nowadays maintained by another service of DANS called NARCIS, which is a research information system.

DANS hosts data archives of other scientific institutions based on bilateral agreements and provides a self-archiving service for the individual researcher. All archived datasets are accessible via DANS archiving utility, EASY's web interface. To enable self-archiving, DANS provides detailed instructions on how to deposit data, which metadata to provide, which are preferred file formats, what access rights options are available, and so on. Users are also asked to choose one or more audiences for the dataset. This information,

\footnotetext{
$\overline{{ }^{4} \text { https://easy.dans.knaw.nl/ui/home }}$

${ }^{5} \mathrm{http}: / /$ www.deviantart.com
}

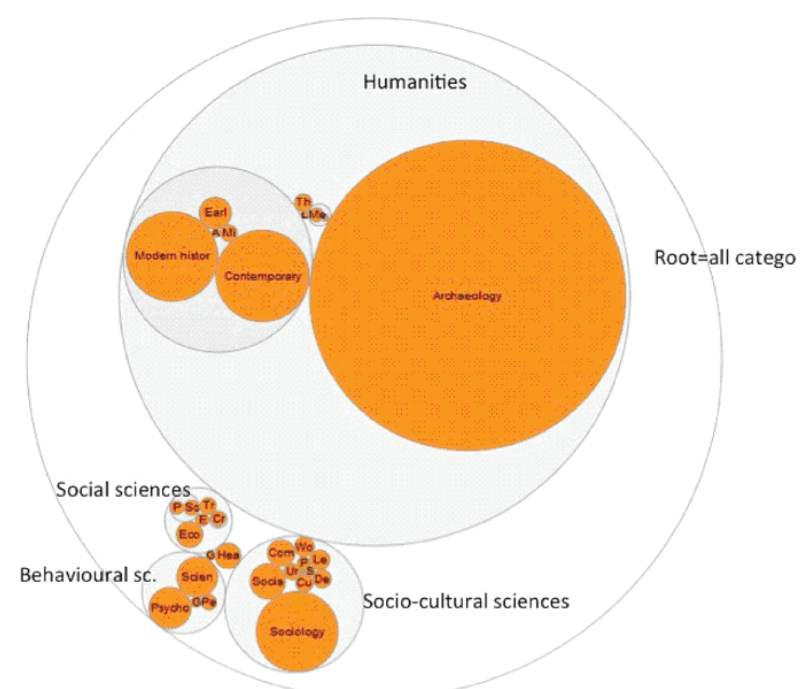

Figure 1: Size of categories in EASY (EASY I)

used to tag the datasets with categories pointing to scientific fields, is meant to stimulate re-use of data and to promote the depositing and sharing of new datasets among certain communities. The self-archiving process is self-guided, but an archivist checks each incoming dataset prior to its publication.

\subsection{Visualization of EASY data}

Two data sets from DANS have been used in this study. DANS provided a dump of the EASY metadata content on November 25, 2011, together with a description of the hierarchical classification of categories used to organize the datasets (EASY I set). There were 19.659 datasets in this set. The total number of unique categories referred to by these datasets was 47. EASY supports the Open Archives Initiative Protocol for Metadata Harvesting (OAI-PMH), through which we extracted a second set of metadata from EASY on January 20, 2012 (EASY II set). The resulting metadata file contained 21.303 datasets. About $70 \%$ of these belong to the Archaeology sub-category. The five main categories currently used are: Humanities (of which Archaeology is a sub-category), Social sciences, Behavioural sciences, Socio-cultural sciences, Life sciences and Medicine (see Figs. 1 \& 2).

The EASY categories are hierarchically organized into a tree, with a maximum depth of three nodes, where leaf nodes can be at any level. Categories can be parents as well as leaf nodes. About $80 \%$ of all datasets are allocated to one category only. A maximum of nine categories are used to tag any single dataset.

We created an interactive treemap, where all datasets with more than one category tag are displayed according to the number of attached categories. This treemap is combined with the visualization of the category tree, on which the size of the category is indicated by a transparent size-scaled circle. Mousing over datasets on the treemap highlights their allocation on the tree. Additionally, the name of the dataset is displayed in a footer below, and clicking on the dataset leads to the original dataset in the archive (Fig. 2) ${ }^{6}$.

\footnotetext{
${ }^{6}$ Snapshot from http://drasticdata.nl/DDHome.php? $\mathrm{m}=514$
} 


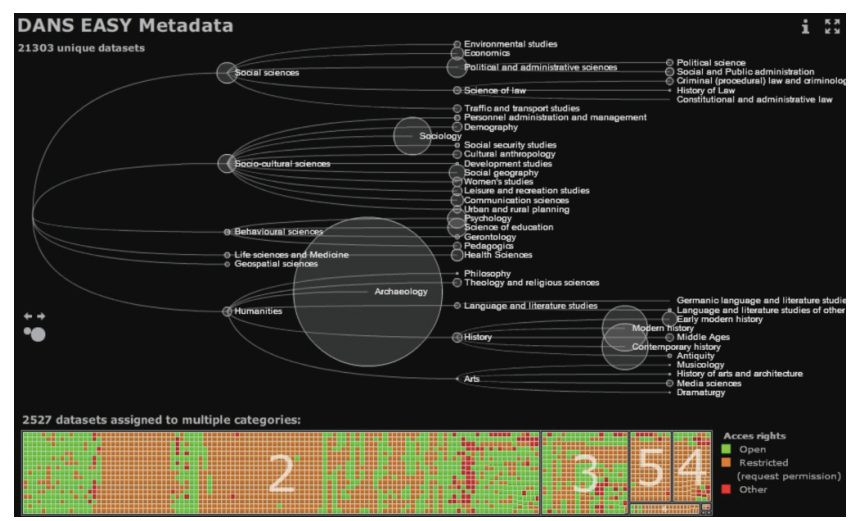

Figure 2: A combination of category tree and treemap in a visual exploration (EASY II).

An analysis of both datasets with specific questions such as: "Who are the key-depositors of EASY?", and "Which datasets are access-controlled?" not only reveals new information, but also emphasizes the relevance of visualization practices in policy making processes. For example, our analysis shows that except Archeology datasets, the disciplinary approach to data sharing is positive. The depositors, as well as the downloaders are important stakeholders for DANS' future.

\section{CASE STUDY 2: DEVIANTART}

\section{1 deviantArt: The largest art online plat- form}

deviantArt $(\mathrm{dA})$ is the largest online community dedicated to arts, with close to 22 million members (as of June 2012), an image archive of about 224 million works, and a number of daily visitors that vastly exceeds any renowned museum [1]. As an SNS, dA offers many services to its members, and enables a platform for sharing not just artworks, but also ideas, techniques, tutorials, reusable images, etc. Similar to other SNS, dA has a lively social life, and the members connect through various channels such as messaging, chatting, commenting. But the main community structure of $\mathrm{dA}$ is based on its category system: each artwork that is uploaded into the $\mathrm{dA}$ has to be put under one, and only one category. A quick look at the categories reveal that the category structure is built predominantly on production techniques (like digital art, traditional art, photography, arts \& crafts, film, animation, etc.) as opposed to genres, and quite naturally, people using similar techniques form social clusters and communities [2].

Every day, deviantArt announces 20-30 artworks as 'Daily Deviations' (DD), and promotes these via the main webpage of $\mathrm{dA}$. The selected works illustrate a representative distribution of the dA community: they might belong to the earliest or latest members, to popular or unknown members [1]. Our data stem from these DD's and galleries of the corresponding artists, acquired directly from dA Headquarters. This data set is composed of members who have received at least one DD, and all members who are following these members, or have favored one of their works. The DDs data set consists of 30.643 daily deviations created by 21.745 deviants between 2000-2011. These deviants had a total of 1.321.264

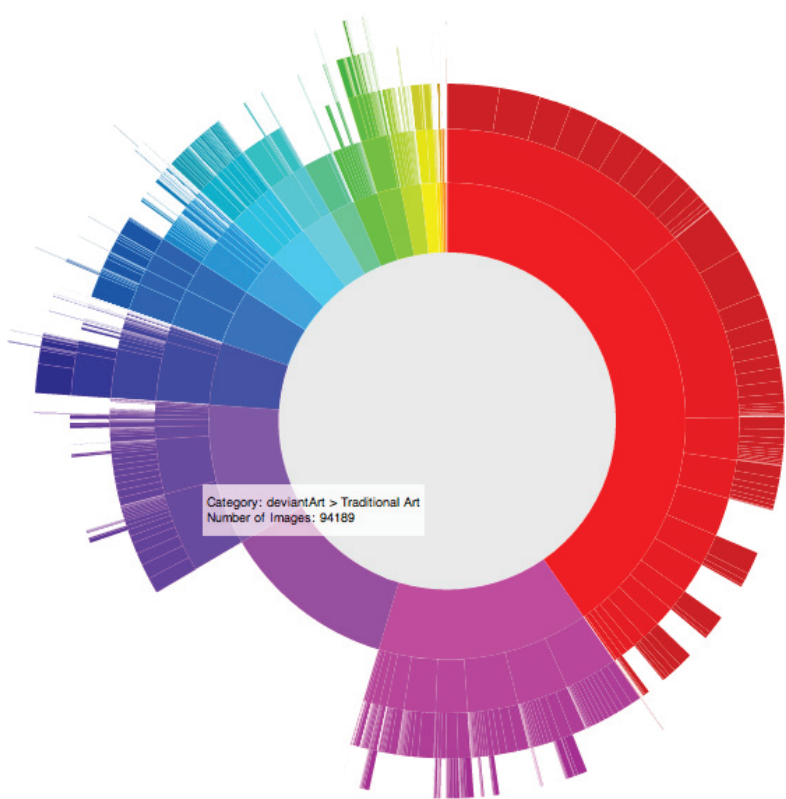

Figure 3: The category structure of dA, based on the DDs dataset.

artworks in dA archive at the time of data retrieval. Beside the artworks themselves, we store category information of the artworks, as well as some basic demographic information about the artists such as age, gender, location (this information is only available if the user chooses to share it).

\subsection{Visualization of deviantArt data}

We have prepared an interactive visualization of the category structure of dA based on the DDs dataset (Fig. 3). We prefer the DDs to the entire set, because it is representative and manageable. This visualization gives an overview of the category structure, and stresses the hierarchical relations between categories. In a related visualization, Akdag Salah et al. have used the number of artists publishing within multiple categories as a measure of the closeness between these categories, and visualized the category structure in 3D [2]. An energy minimization procedure reveals which categories are perused by the same artists by bringing the corresponding nodes closer in space; for instance people photography and nature photography have a lot of overlapping artists, but so does traditional manga and digital manga, which is much more surprising. The visualization of [2] displays these relations, yet loses the hierarchy completely, which is relevant for the artist who seeks to position his or her work within the archive.

The most occupied category of dA is photography, followed by digital art, and then traditional art ${ }^{7}$. It is interesting to see that the most occupied category is the one with least depth going down the category structure. In contrast,

\footnotetext{
${ }^{7}$ We disregard the category of scraps even though it is included in the visualization. This category is used as a work in progress by the members, and hence the artworks under this category do not have subcategories, and are not directly related with the rest of the category tree in an ontological manner. The interactive visualization is at http://neen.ucsd.edu/DeviantArt/ Visualizations/d3vis/ sunburst/category.html
} 
the smallest top categories like fan art or manga art have an intricate category structure, with subcategories down to the $6^{\text {th }}$ level. The reason of this rather unusual distribution of subcategories might be due to the differences in the practices of photography versus other techniques. The existing structure not only stems from a natural clustering of genre and technique, but may point out to trends and sub-communities. It is also interesting to investigate the evolution of the proposed visualization over time.

The exploration of DD dataset's category structure reveals interesting patterns, of significance from both a research point of view, as well as from a commercial point of view. Even though the category structure plays a major role as the backbone of the community, in practice, providing a purely category-based search experience, with the interactive overview chart that we have prepared, would have little to give to the end-users, i.e. the $\mathrm{dA}$ members. Browsing artworks is a very subjective practice, and dA has carefully avoided recommender schemes based on user similarity since its inception. Of course a user might want to know how many members there are, working on a certain subcategory, or who the most productive members of a category are, or which members of a certain age/location are producing more under a given category. Even though dA is not strictly a professional art market (its membership is free, and admits amateurs as well as professionals), still it has an interface for selling artworks, used by many members who regard $\mathrm{dA}$ as more than a free-time occupation. For such users, it would be important to target the right audience, and to know more about dA structure to reach out to this audience.

Given the richness of the archive of dA (which includes artworks, descriptions on artworks, demographic information, member interactions, pageview statistics, comments on arts and artists, etc.) the combinations of visualizations are endless. Especially vital would be to use real-time network visualizations (i.e. showing which member is following which categories/groups, who is connected to whom through what type of relation ${ }^{8}$. A combination of such network visualizations with an overview of the collection according to category structure would enhance the user's understanding of his/her position in this social network and help him/her to find vital signposts (i.e. hubs, authorities, gate holders).

\section{CONCLUSIONS}

In this paper we emphasize the need for interactive visualizations for accessing large data collections, as well as stress that there may not be a single software solution that would generically solve all visualization problems, since different social networks will have different information demands.

Obviously, dA presents an entirely different case study where users would indeed benefit from an exploration of the different parts of the network in addition to the immediate surroundings of their own environment. The social life of dA is not limited to following each others activities and commenting on these; it is full with community activities such as contests, news, announcements, polls, etc. It would be another challenge to visualize how members are reacting to such activities, i.e. how many members respond by leaving comments, joining the contests, announcements, etc. Realtime, interactive visualizations of social networking sites is

\footnotetext{
${ }^{8}$ In dA, members can become 'friends', give comments, or watch each other to receive activity notifications.
}

not a new idea [6], however, a working solution is not offered yet.

Our explorations point out to the fact that category structure is an essential feature of the metadata of many archives and should not be neglected when developing visual mechanisms. There is an important difference between providing a classification structure and using it for indexing purposes. The way how categories are applied in tagging creates an alternative network of relations between fields.

The presented analysis of EASY was also an experiment towards the development of visual facetted and visual enhanced browsing. For this reason we have not only analyzed and visualized the data, but published several interactive displays for DANS-EASY on the web. The visualization of datasets across categories, as well as across categories and creators entail a search interface ${ }^{9}$. This search function is much more rudimentary than the one used in the interface of EASY. However, it indicates how usual text-based search can be combined with visual exploration.

\section{ACKNOWLEDGMENTS}

This research is supported by an NWO-Veni grant (Akdag Salah) and as a KDP (Klein Data Projekt) of DANS. Special thanks to Henk Koning (DANS) for the EASY I data set. We are grateful for comments and inspiring discussions with Katy Börner (SLIS, Indiana University, USA), Henk Koning and Linda Reijnhoudt (DANS).

\section{ADDITIONAL AUTHORS}

Additional authors: Jay Chow (Software Studies, UCSD email: jac035@ucsd.edu) .

\section{REFERENCES}

[1] A. A. Akdag Salah. The online potential of art creation and dissemination: deviantART as the next art venue. Proc. Electronic Visualisation and the Arts, 2010.

[2] A. A. Akdag Salah, A. A. Salah, B. Buter, N. Dijkshoorn, D. Modolo, Q. Nguyen, S. van Noort, and B. van de Poel. DeviantArt in spotlight: A network of artists. Leonardo, 45(5), 2012.

[3] C. Borgman. Why are online catalogs still hard to use? Journal of the American Society for Information Science, 47(7):493-503, 1996.

[4] K. Börner and C. Chen. Visual Interfaces to Digital Libraries: Motivation, Utilization, and Socio-technical Challenges. LNCS. Springer, 2002.

[5] M. Breeding. AquaBrowser. Library Technology Reports, 43(4):15-18, 2007.

[6] J. Heer and D. Boyd. Vizster: Visualizing online social networks. In INFOVIS, pages 32-39, 2005.

[7] B. Shneiderman, D. Feldman, A. Rose, and X. Grau. Visualizing digital library search results with categorical and hierarchical axes. In Proc. ACM Conf. on Digital libraries, pages 57-66, 2000.

[8] F. Viégas, M. Wattenberg, and J. Feinberg. Participatory visualization with Wordle. IEEE Trans. Visualization and Computer Graphics, 15(6):1137-1144, 2009.

\footnotetext{
${ }^{9}$ http://drasticdata.nl/ProjectDANSEasy
} 